\title{
The rational homology of spaces of long links
}

\author{
Paul ARnAud Songhafouo TsopmÉnÉ
}

\begin{abstract}
We provide a complete understanding of the rational homology of the space of long links of $m$ strands in $\mathbb{R}^{d}$ when $d \geq 4$. First, we construct explicitly a cosimplicial chain complex, $L_{*}^{\bullet}$, whose totalization is quasi-isomorphic to the singular chain complex of the space of long links. Next we show, using the fact that the BousfieldKan spectral sequence associated to $L_{*}^{\bullet}$ collapses at the $E^{2}$ page, that the homology Bousfield-Kan spectral sequence associated to the Munson-Volić cosimplicial model for the space of long links collapses at the $E^{2}$ page rationally, solving a conjecture of B Munson and I Volić. Our method enables us also to determine the rational homology of high-dimensional analogues of spaces of long links. Our last result states that the radius of convergence of the Poincaré series for the space of long links (modulo immersions) tends to zero as $m$ goes to infinity.
\end{abstract}

57Q45; 18D50, 18G40, 55P48

\section{Introduction}

A long link of $m$ strands in $\mathbb{R}^{d}, d \geq 3$, is a smooth embedding $\bigsqcup_{1}^{m} \mathbb{R} \hookrightarrow \mathbb{R}^{d}$ of $m$ copies of $\mathbb{R}$ inside $\mathbb{R}^{d}$, which coincides outside a compact set with a fixed standard embedding. Such embedding is said to be compactly supported. We denote by $\operatorname{Emb}_{c}\left(\bigsqcup_{1}^{m} \mathbb{R}, \mathbb{R}^{d}\right)$ or simply by $\mathcal{L}_{m}^{d}$ the space of long links of $m$ strands, and define the space $\operatorname{Imm}_{c}\left(\bigsqcup_{1}^{m} \mathbb{R}, \mathbb{R}^{d}\right)$ of long immersions of $m$ strands analogously. It is clear that there is an inclusion $\operatorname{Emb}_{c}\left(\bigsqcup_{1}^{m} \mathbb{R}, \mathbb{R}^{d}\right) \hookrightarrow \operatorname{Imm}_{c}\left(\bigsqcup_{1}^{m} \mathbb{R}, \mathbb{R}^{d}\right)$, whose homotopy fiber is called the space of long links modulo immersions, and is denoted by $\overline{\operatorname{Emb}}_{c}\left(\bigsqcup_{i=1}^{m} \mathbb{R}, \mathbb{R}^{d}\right)$ or simply by $\overline{\mathcal{L}}_{m}^{d}$. This paper is devoted to the study of the latter space. More precisely, we completely determine the rational homology of $\overline{\mathcal{L}}_{m}^{d}$. In fact, we prove that the homology Bousfield-Kan spectral sequence associated to the Munson-Volić cosimplicial model for $\overline{\mathcal{L}}_{m}^{d}$ collapses at the $E^{2}$ page rationally.

Let $\operatorname{Conf}\left(k, \mathbb{R}^{d}\right)$ denote the space of configuration of $k$ points in $\mathbb{R}^{d}$. We will construct an explicit cosimplicial chain complex $L_{*}^{\bullet}$, where

$$
L_{*}^{p}=H_{*}\left(\operatorname{Conf}\left(m p, \mathbb{R}^{d}\right) ; \mathbb{Q}\right) .
$$


Let $B_{d}$ denote the little $d$-disks operad, and let $s^{-p}$ be the suspension functor of degree $-p$. Define the totalization Tot $L_{*}^{\bullet}$ to be

$$
\operatorname{Tot} L_{*}^{\bullet}=\bigoplus_{p \geq 0}\left(s^{-p} L_{*}^{p}\right)
$$

where the differential is the alternate sum of cofaces. We will show in this introduction that the homology of this totalization can be interpreted by the $\bigvee_{i=1}^{m} S^{1}$-homology of $H_{*}\left(B_{d} ; \mathbb{Q}\right)$.

Our first result says that the cosimplicial chain complex $L_{*}^{\bullet}$ gives a cosimplicial model for the singular chain complex of the space of long links.

Theorem 1.1 For $d \geq 4$, the totalization of $L_{*}^{\bullet}$ is quasi-isomorphic to the chain complex of the space of long links of $m$ strands in $\mathbb{R}^{d}$. That is,

$$
\operatorname{Tot} L_{*}^{\bullet} \simeq C_{*}\left(\overline{\mathcal{L}}_{m}^{d}\right) \otimes \mathbb{Q} \text {. }
$$

The following corollary is an immediate consequence of Theorem 1.1.

Corollary 1.2 For $d \geq 4$, the rational homology of the space of long links of $m$ strands is isomorphic to the $\bigvee_{i=1}^{m} S^{1}-$ homology of $H_{*}\left(B_{d} ; \mathbb{Q}\right)$. That is,

$$
H_{*}\left(\overline{\mathcal{L}}_{m}^{d} ; \mathbb{Q}\right) \cong H H^{\vee_{i=1}^{m} S^{1}}\left(H_{*}\left(B_{d} ; \mathbb{Q}\right)\right) .
$$

We now explain what we mean by the $\bigvee_{i=1}^{m} S^{1}$-homology of $H_{*}\left(B_{d} ; \mathbb{Q}\right)$. Let $\Gamma$ denote the category of finite pointed sets whose morphisms are maps preserving the base point. If $X$. is a simplicial object in $\Gamma$, and if $A$ is a contravariant functor from $\Gamma$ to chain complexes, then the composite $A\left(X_{\bullet}\right): \Delta \longrightarrow \mathrm{Ch}_{*}$ yields a cosimplicial chain complex, and the homology of its totalization, denoted by $H H^{X}(A)$, is called the $X$-homology of $A$. Here, the simplicial model $\left(\bigvee_{i=1}^{m} S^{1}\right)$. (the one we construct at the beginning of Section 4 ) of the wedge of $m$ copies of the circle is viewed as a simplicial object in $\Gamma$, while the homology $H_{*}\left(B_{d} ; \mathbb{Q}\right): \Gamma \longrightarrow \mathrm{Ch}_{*}$ is viewed as a contravariant functor from $\Gamma$ to chain complexes. Hence, the composite $H_{*}\left(B_{d}, \mathbb{Q}\right)\left(\left(\bigvee_{i=1}^{m} S^{1}\right)\right.$.) gives another way to see the cosimplicial chain complex $L_{*}^{\bullet}$, so that we can set

$$
L_{*}^{\bullet}:=H_{*}\left(B_{d}, \mathbb{Q}\right)\left(\left(\bigvee_{i=1}^{m} S^{1}\right)_{\bullet}\right) \text {. }
$$

We now state the second and the most important result of this paper, which solves a conjecture of Munson and Volić. In [9], Munson and Volić build a cosimplicial space (we denote it by $\mathcal{K}_{d}^{m \bullet}$ ) that gives a cosimplicial model for the space $\overline{\mathcal{L}}_{m}^{d}$ of long links of $m$ strands in $\mathbb{R}^{d}$, when $d \geq 4$. They also define two spectral sequences that converge respectively to the homotopy and cohomology of the space $\overline{\mathcal{L}}_{m}^{d}$ of long links. In this 
paper, we look at the homology Bousfield-Kan spectral sequence associated to $\mathcal{K}_{d}^{m \bullet}$, which converges to the homology $H_{*}\left(\overline{\mathcal{L}}_{m}^{d}\right)$ (this is a consequence of Theorem 1.1).

Conjecture 1.3 (Munson-Volić) This spectral sequence collapses at the $E^{2}$ page rationally for $d \geq 4$.

Theorem 1.4 For $d \geq 4$, the homology Bousfield-Kan spectral sequence associated to the Munson-Volić cosimplicial model $\mathcal{K}_{d}^{m \bullet}$ for the space of long links of $m$ strands in $\mathbb{R}^{d}$ collapses at the $E^{2}$ page rationally.

Remark 1.5 Our method enables us also to determine the rational homology of high-dimensional analogues of spaces $\overline{\operatorname{Emb}}_{c}\left(\bigsqcup_{i=1}^{m} \mathbb{R}^{n}, \mathbb{R}^{d}\right)$ of long links modulo immersions. More precisely, as in the case of long links, we construct an explicit cosimplicial chain complex $L_{*}^{n \bullet}$ and we prove that it gives a cosimplicial model for the singular chain complex of $\overline{\operatorname{Emb}}_{c}\left(\bigsqcup_{i=1}^{m} \mathbb{R}^{n}, \mathbb{R}^{d}\right)$. Thus we obtain Theorem 5.2, Corollary 5.3 and Proposition 5.4. With the same strategy, one can go further by understanding the rational homology of $\overline{\operatorname{Emb}}_{c}\left(\bigsqcup_{i=1}^{m} \mathbb{R}^{n_{i}}, \mathbb{R}^{d}\right)$ for any integers $n_{i} \geq 1$.

The case $m=1$ (this case corresponds to $\overline{\mathrm{Emb}}_{c}\left(\mathbb{R}, \mathbb{R}^{d}\right.$ ), the space of long knots modulo immersions) has been studied recently by several authors. First, Sinha constructs in [11] a cosimplicial model $\mathcal{K}_{d}^{\bullet}$ of $\overline{\mathrm{Emb}}_{c}\left(\mathbb{R}, \mathbb{R}^{d}\right)$ when $d \geq 4$. Next, Lambrechts, Turchin and Volić prove [5] that the homology Bousfield-Kan spectral sequence associated to $\mathcal{K}_{d}^{\bullet}$ collapses at the $E^{2}$ page rationally when $d \geq 4$. Subsequently, the author [12] and Moriya [8] prove independently that the collapsing result still holds for $d \geq 3$, and simplify the proof of the main result of [5]. In this paper, we produce (using a completely different approach than that of $[5 ; 8 ; 12])$ another proof of the collapsing result.

Other interesting results obtained in the study of the space of long knots are the following. The author [12] and Moriya [8] independently discover the multiplicative formality (for $d \geq 3$ ) of the Kontsevich operad $\mathcal{K}_{d}(\bullet)$. This result has two strong consequences: the first one is immediate and says that Sinha's cosimplicial space $\mathcal{K}_{d}^{\bullet}$ is formal when $d \geq 3$. The second and most important one furnishes a complete understanding of the rational homology of the space of long knots as a Gerstenhaber algebra. In fact, this second consequence states [12, Corollary 1.6] that, for $d \geq 4$, the isomorphism of vector spaces between the $E^{2}$ page and the homology $H_{*}\left(\overline{\mathrm{Emb}}_{c}\left(\mathbb{R}, \mathbb{R}^{d}\right)\right)$ of the space of long knots (modulo immersions) respects the Gerstenhaber algebra structure.

We close this introduction with our last result, which concerns the Poincaré series for the space of long links. In [4] Komawila and Lambrechts study the $E^{2}$ page of the cohomology Bousfield-Kan spectral sequence associated to the Munson-Volić 
cosimplicial space. They show that the coefficients of the associated Euler series grow exponentially at a rate of $m^{1 /(d-1)}>1$. Using now our collapsing Theorem 1.4 , we deduce the following result.

Theorem 1.6 For $d \geq 4$ the radius of convergence of the Poincaré series for the space of long links (modulo immersions) $\overline{\mathcal{L}}_{m}^{d}$ is less than or equal to $(1 / m)^{1 /(d-1)}$. Therefore the Betti numbers of $\overline{\mathcal{L}}_{m}^{d}$ grow at least exponentially.

An immediate consequence of Theorem 1.6 is the following corollary.

Corollary 1.7 For $d \geq 4$ the radius of convergence of the Poincare series for $\overline{\mathcal{L}}_{m}^{d}$ tends to 0 as $m$ goes to $\infty$.

When $m=1$ the upper bound of Theorem 1.6 is equal to 1 , and the following theorem, due to Turchin, gives a better upper bound in that case.

Theorem 1.8 [14] For $d \geq 4$ the radius of convergence of the Poincaré series for the space of long knots (modulo immersions) is less than or equal to $(1 / \sqrt{2})^{1 /(d-1)}$.

Since the space of $m$ copies of long knots is a retraction up to homotopy of the space of long links, Theorem 1.8 implies that the radius of convergence of the Poincaré series for $\overline{\mathcal{L}}_{m}^{d}$ is less than or equal to $(1 / \sqrt{2})^{1 /(d-1)}$. Our Corollary 1.7 furnishes a better upper bound for $m$ large.

Outline of the paper In Section 2 we set up some results that we will use to prove Theorem 1.1 and Theorem 1.4. First of all, we define a manifold $M$, and we show that (see Proposition 2.3 below) the study of the space of long links modulo immersions is reduced to the study of the space $\overline{\operatorname{Emb}}_{c}\left(M, \mathbb{R}^{d}\right)$ of compactly supported embeddings of $M$ into $\mathbb{R}^{d}$. Next we recall some results, related to the Taylor tower associated to $\overline{\mathrm{Emb}}_{c}\left(\mathbb{R}^{n}, \mathbb{R}^{d}\right)$, obtained by Arone and Turchin in [1]. Finally, we show that similar results (Propositions 2.9, 2.10, 2.11 and 2.12) hold for the space $\overline{\operatorname{Emb}}_{c}\left(M, \mathbb{R}^{d}\right.$ ).

In Section 3 we construct an explicit cosimplicial chain complex $L_{*}^{\bullet}$ that gives a cosimplicial model for the singular chain complex $C_{*}\left(\overline{\operatorname{Emb}}_{c}\left(M, \mathbb{R}^{d}\right) ; \mathbb{Q}\right)$ (Theorem 1.1). To prove Theorem 1.1, we use all the results from Section 2.2, and also Proposition 3.4, Lemma 3.5 and Theorem 3.6.

In Section 4 we prove Theorem 1.4. The plan of the proof is as follows. First we build a simplicial model for the wedge of $m$ copies of the circle. Next we prove (Lemma 4.2) that the $E^{1}$ pages of spectral sequences $\left\{E^{r}\left(L_{*}^{\bullet}\right)\right\}_{r \geq 0}$ and $\left\{E^{r}\left(C_{*}\left(\mathcal{K}_{d}^{m \bullet} ; \mathbb{Q}\right)\right)\right\}_{r \geq 0}$ are 
isomorphic. Using now the fact that the spectral sequence $\left\{E^{r}\left(L_{*}^{\bullet}\right)\right\}_{r \geq 0}$ collapses at the $E^{2}$ page (Lemma 4.3) and Theorem 1.1, we deduce Theorem 1.4.

In Section 5 we show that the spectral sequence computing the rational homology of the high-dimensional analogues of spaces of long links collapses at the $E^{2}$ page.

In Section 6 we show that the radius of convergence of the Poincaré series for the space of long links modulo $m$ copies of the space of long knots tends to zero as $m$ goes to the infinity. This result is obtained from Theorem 1.4 and a theorem of Komawila and Lambrechts [4].

Acknowledgements I am grateful to my advisor Pascal Lambrechts for his outstanding guidance on this work and also for his encouragement. I also thank Gregory Arone for his valuable help with Proposition 2.3 (which is one of the central observations in this paper) and for helpful conversations (by emails) that we have had during the preparation of the first draft of this paper. Obviously, I cannot forget to thank Victor Turchin for his explanation on infinitesimal bimodules and for suggesting the statement of Proposition 3.4.

\section{A compactly supported version of Goodwillie-Weiss em- bedding calculus for the space of long links}

We introduce this section with Proposition 2.3, which allows us to reduce the study of the space $\overline{\mathcal{L}}_{m}^{d}$ of long links to the study of the space $\overline{\operatorname{Emb}}_{c}\left(M, \mathbb{R}^{d}\right)$ of compactly supported embeddings of some manifold $M$ into $\mathbb{R}^{d}$. Before stating that proposition, we will properly define $M$ and the spaces $\overline{\mathcal{L}}_{m}^{d}$ and $\overline{\operatorname{Emb}}_{c}\left(M, \mathbb{R}^{d}\right)$. The ground field in this section is $\mathbb{Q}$.

Roughly speaking, $M$ is the complement in $\mathbb{R}^{2}$ of a slightly thickening of $m+1$ copies of the interval $I=[-1,1]$. To be more precise, let $\left\{a_{0}, a_{1}, \ldots, a_{m}\right\} \subseteq I$ be the family of points defined by

$$
a_{i}=\frac{2 i-m-1}{m+1} .
$$

Let $0<\epsilon<\frac{2}{m+1}$ be a fixed real number. For $0 \leq i \leq m$, define

$$
K_{i}= \begin{cases}I \times\left[a_{i}, a_{i}+\epsilon\right] & \text { if } 0 \leq i \leq m-1 \\ I \times\left[a_{m}, 1\right] & \text { if } i=m\end{cases}
$$

Definition 2.1 Let $M$ be the complement of $K=\bigcup_{i=0}^{m} K_{i}$ in $\mathbb{R}^{2}$. That is,

$$
M=\mathbb{R}^{2} \backslash \bigcup_{i=0}^{m} K_{i} .
$$


We now define the spaces $\overline{\mathcal{L}}_{m}^{d}$ and $\overline{\operatorname{Emb}}_{c}\left(M, \mathbb{R}^{d}\right)$. Let $\eta: \mathbb{R}^{2} \hookrightarrow \mathbb{R}^{d}$ be a fixed linear embedding defined by $\eta(t, x)=(0, \ldots, 0, t, x)$. Define $\operatorname{Emb}_{c}\left(M, \mathbb{R}^{d}\right)$ to be the space of smooth embeddings $f: M \hookrightarrow \mathbb{R}^{d}$ such that

$$
f(t, x)=\eta(t, x) \quad \text { for all }(t, x) \notin]-1,1[\times]-1,1[.
$$

This space is equipped with the weak $C^{\infty}$-topology. Furthermore, define the space $\operatorname{Emb}_{c}\left(\bigsqcup_{i=1}^{m} \mathbb{R}, \mathbb{R}^{d}\right)$ of smooth embeddings $f: \mathbb{R} \times\left\{b_{1}, \ldots, b_{m}\right\} \hookrightarrow \mathbb{R}^{d}$ such that $f_{i}(t)=\eta\left(t, b_{i}\right)$ for all $|t| \geq 1$ and for all $1 \leq i \leq m$. Here $b_{i}=\frac{1}{2}\left(a_{i-1}+\epsilon+a_{i}\right)$ and $f_{i}$ is the restriction of $f$ to $\mathbb{R} \times\left\{b_{i}\right\}$. Similarly, we have the $\operatorname{spaces} \operatorname{Imm}_{c}\left(M, \mathbb{R}^{d}\right)$ and $\operatorname{Imm}_{c}\left(\bigsqcup_{i=1}^{m} \mathbb{R}, \mathbb{R}^{d}\right)$ of compactly supported immersions. By definition, there are inclusions

$\operatorname{Emb}_{c}\left(M, \mathbb{R}^{d}\right) \hookrightarrow \operatorname{Imm}_{c}\left(M, \mathbb{R}^{d}\right) \quad$ and $\quad \operatorname{Emb}_{c}\left(\bigsqcup_{i=1}^{m} \mathbb{R}, \mathbb{R}^{d}\right) \hookrightarrow \operatorname{Imm}_{c}\left(\bigsqcup_{i=1}^{m} \mathbb{R}, \mathbb{R}^{d}\right)$.

Let $l \in \operatorname{Imm}_{c}\left(M, \mathbb{R}^{d}\right)$ be a fixed immersion, and let $\tilde{l} \in \operatorname{Imm}_{c}\left(\bigsqcup_{i=1}^{m} \mathbb{R}, \mathbb{R}^{d}\right)$ be the restriction of $l$ to $\bigsqcup_{i=1}^{m} \mathbb{R} \times\left\{b_{i}\right\}$.

Definition 2.2 (i) The space $\overline{\operatorname{Emb}}_{c}\left(M, \mathbb{R}^{d}\right)$ is the homotopy pullback of the diagram

$$
\{l\} \hookrightarrow \operatorname{Imm}_{c}\left(M, \mathbb{R}^{d}\right) \hookleftarrow \operatorname{Emb}_{c}\left(M, \mathbb{R}^{d}\right) .
$$

(ii) The space $\overline{\operatorname{Emb}}_{c}\left(\bigsqcup_{i=1}^{m} \mathbb{R}, \mathbb{R}^{d}\right)$ or more simply $\overline{\mathcal{L}}_{m}^{d}$ is the homotopy pullback of the diagram

$$
\{\tilde{l}\} \hookrightarrow \operatorname{Imm}_{c}\left(\bigsqcup_{i=1}^{m} \mathbb{R}, \mathbb{R}^{d}\right) \hookleftarrow \operatorname{Emb}_{c}\left(\bigsqcup_{i=1}^{m} \mathbb{R}, \mathbb{R}^{d}\right) .
$$

The following proposition is a central observation in this paper.

Proposition 2.3 For $d \geq 3$, the space of long links modulo immersions in $\mathbb{R}^{d}$ is weakly equivalent to the space of smooth compactly supported embeddings (modulo immersions) of $M$ in $\mathbb{R}^{d}$. That is,

$$
\overline{\mathcal{L}}_{m}^{d} \simeq \overline{\operatorname{Emb}}_{c}\left(M, \mathbb{R}^{d}\right) .
$$

Proof For $1 \leq i \leq m$, set $\left.A_{i}=I \times\right] a_{i-1}+\epsilon, a_{i}\left[\right.$, and define $\operatorname{Emb}_{c}\left(\bigsqcup_{i=1}^{m} A_{i}, I^{d}\right)$ to be the space of smooth embeddings

$$
f: \bigsqcup_{i=1}^{m} A_{i} \hookrightarrow I^{d}
$$


satisfying the boundary conditions

$$
f(-1, x)=\eta(-1, x) \quad \text { and } \quad f(1, x)=\eta(1, x)
$$

for all $x \in] a_{i-1}+\epsilon, a_{i}[, 1 \leq i \leq m$, and

$$
T_{f(-1, x)} f\left(A_{i}\right)=T_{f(-1, x)} \eta\left(A_{i}\right) \quad \text { and } \quad T_{f(1, x)} f\left(A_{i}\right)=T_{f(1, x)} \eta\left(A_{i}\right)
$$

for all $1 \leq i \leq m$ and for all $x \in] a_{i-1}+\epsilon, a_{i}\left[\right.$. Here $T_{p} X$ denotes the tangent space of $X$ at $p$.

Define also $\operatorname{Emb}_{c}\left(\bigsqcup_{i=1}^{m} I, I^{d}\right)$ to be the space of smooth embeddings

$$
f: \bigsqcup_{i=1}^{m} I \times\left\{b_{i}\right\} \hookrightarrow I^{d}
$$

satisfying similar boundary conditions as above. That is, the endpoints of $f$ and the tangent vectors at those endpoints are given by $\eta$. We define similarly the spaces $\operatorname{Imm}_{c}\left(\bigsqcup_{i=1}^{m} A_{i}, I^{d}\right)$ and $\operatorname{Imm}_{c}\left(\bigsqcup_{i=1}^{m} I, I^{d}\right)$. As in Definition 2.2, we have the spaces $\overline{\operatorname{Emb}}_{c}\left(\bigsqcup_{i=1}^{m} A_{i}, I^{d}\right)$ and $\overline{\operatorname{Emb}}_{c}\left(\bigsqcup_{i=1}^{m} I, I^{d}\right)$ (the fixed immersions here are, respectively, the restrictions of $l$ to $\bigsqcup_{i=1}^{m} A_{i}$ and to $\left.\bigsqcup_{i=1}^{m} I \times\left\{b_{i}\right\}\right)$. From the definitions, one can easily see the weak equivalences

$$
\overline{\mathcal{L}}_{m}^{d} \simeq \overline{\operatorname{Emb}}_{c}\left(\bigsqcup_{i=1}^{m} I, I^{d}\right) \text { and } \overline{\operatorname{Emb}}_{c}\left(M, \mathbb{R}^{d}\right) \simeq \overline{\operatorname{Emb}}_{c}\left(\bigsqcup_{i=1}^{m} A_{i}, I^{d}\right) .
$$

To end the proof, it suffices to show that there is a weak equivalence

$$
\overline{\operatorname{Emb}}_{c}\left(\bigsqcup_{i=1}^{m} I, I^{d}\right) \simeq \overline{\operatorname{Emb}}_{c}\left(\bigsqcup_{i=1}^{m} A_{i}, I^{d}\right) \text {. }
$$

Notice that the spaces $\operatorname{Emb}_{c}\left(\bigsqcup_{i=1}^{m} A_{i}, I^{d}\right)$ and $\operatorname{Emb}_{c}\left(\bigsqcup_{i=1}^{m} I, I^{d}\right)$ are not weakly equivalent, for the following reason. For an element $f$ in the first space, we have the tangent space (which is a 2-dimensional vector space) at each point $f(t, x)$, while at each point $g(t)$ of the second space, where $g$ is the restriction of $f$ to $\bigsqcup_{i=1}^{m} I \times\left\{b_{i}\right\}$, we have a 1-dimensional vector space as the tangent space. For the homotopy fibers $\overline{\mathrm{Emb}}_{c}\left(\bigsqcup_{i=1}^{m} I, I^{d}\right)$ and $\overline{\mathrm{Emb}}_{c}\left(\bigsqcup_{i=1}^{m} A_{i}, I^{d}\right)$, this tangential information disappears because of the prescribed immersion $l$. We thus obtain (2).

Remark 2.4 The idea of Proposition 2.3 (which consists in studying $\overline{\operatorname{Emb}}_{c}\left(M, \mathbb{R}^{d}\right.$ ) instead of $\overline{\mathcal{L}}_{m}^{d}$ ) can be generalized to the study of the rational homology of the space $\overline{\mathrm{Emb}}_{c}\left(\bigsqcup_{i=1}^{m} \mathbb{R}^{n_{i}}, \mathbb{R}^{d}\right)$ of high string links (here the integers $n_{i}$ are not necessarily the 
same). For example, in the case $n_{i}=n$ for all $i$, which will be studied in Section 5, one can define a submanifold $M_{n} \subseteq \mathbb{R}^{n+1}$ by

$$
M_{n}=\mathbb{R}^{n+1} \backslash \bigcup_{i=0}^{m} K_{i n},
$$

where

$$
K_{i n}= \begin{cases}I^{n} \times\left[a_{i}, a_{i}+\epsilon\right] & \text { if } 0 \leq i \leq m-1, \\ I^{n} \times\left[a_{m}, 1\right] & \text { if } i=m\end{cases}
$$

As a generalization of Proposition 2.3, one can prove the weak equivalence

$$
\overline{\operatorname{Emb}}_{c}\left(\bigsqcup_{i=1}^{m} \mathbb{R}^{n}, \mathbb{R}^{d}\right) \simeq \overline{\operatorname{Emb}}_{c}\left(M_{n}, \mathbb{R}^{d}\right) .
$$

The advantage to working with $\overline{\operatorname{Emb}}_{c}\left(M, \mathbb{R}^{d}\right)$ instead of $\overline{\mathcal{L}}_{m}^{d}$ is that one can use the same techniques as those developed by Arone and Turchin [1] in the study of the space $\overline{\operatorname{Emb}}_{c}\left(\mathbb{R}^{n}, \mathbb{R}^{d}\right)$. They show that the $k^{\text {th }}$ approximation of the Taylor tower associated to the chain complex $C_{*}\left(\overline{\operatorname{Emb}}_{c}\left(\mathbb{R}^{n}, \mathbb{R}^{d}\right)\right)$, that is, the Taylor tower of the functor $V \longmapsto C_{*}\left(\overline{\operatorname{Emb}}_{c}\left(V, \mathbb{R}^{d}\right)\right)$, can be expressed in terms of morphisms between infinitesimal bimodules over the operad $C_{*}\left(B_{n}\right)$. The goal of this section is to show that we obtain results similar to theirs (for the Taylor tower of $\overline{\operatorname{Emb}}_{c}\left(M, \mathbb{R}^{d}\right)$ ). To state and prove our results, it is easiest to first review what is done in [1].

\subsection{Review of the Taylor tower associated to $\overline{\operatorname{Emb}}_{c}\left(\mathbb{R}^{n}, \mathbb{R}^{d}\right)$}

Let $\mathcal{O}\left(\mathbb{R}^{n}\right)$ be the poset of open subsets of $\mathbb{R}^{n}$. Define $\widetilde{\mathcal{O}}\left(\mathbb{R}^{n}\right) \subseteq \mathcal{O}\left(\mathbb{R}^{n}\right)$ to be the subcategory of open subsets whose complement is bounded. Define also the category $\widetilde{\mathcal{O}}_{k}\left(\mathbb{R}^{n}\right)$ to be the subcategory of $\widetilde{\mathcal{O}}\left(\mathbb{R}^{n}\right)$ consisting of disjoint unions $U=U_{0} \cup U_{1}$ such that $U_{0}$ is the complement of a closed ball, and $U_{1}$ is the disjoint union of at most $k$ open balls in $\mathbb{R}^{n}$. Assume that there is an inclusion $\mathbb{R}^{n} \hookrightarrow \mathbb{R}^{d}$. Then one may define a contravariant functor

$$
\overline{\operatorname{Emb}}_{c}\left(-, \mathbb{R}^{d}\right): \widetilde{\mathcal{O}}\left(\mathbb{R}^{n}\right) \longrightarrow \text { Top } .
$$

Taking that functor as an input in a "compactly supported" version of GoodwillieWeiss embedding calculus $[16 ; 3]$, one obtains Proposition 2.5 below, which states that the $k^{\text {th }}$ approximation $T_{k} \overline{\operatorname{Emb}}_{c}\left(\mathbb{R}^{n}, \mathbb{R}^{d}\right)$ can be expressed as the space of maps between infinitesimal bimodules over the little $n$-disks operad $B_{n}$. Before stating that proposition, we recall the definition of an infinitesimal bimodule from [1, Definition 3.8] or from [14, Definition 4.1]. Recall also the following notation. By $\operatorname{InfBim}_{\mathcal{O}}$, we denote the category of infinitesimal bimodules over an operad $\mathcal{O}$, and by $\operatorname{InfBim}_{\mathcal{O}}^{\leq k}$ 
we denote its $k^{\text {th }}$ truncation. If $\mathcal{B}_{1}$ and $\mathcal{B}_{2}$ are two infinitesimal bimodules over $\mathcal{O}$, we denote by $\operatorname{hInfBim}_{\mathcal{O}}\left(\mathcal{B}_{1}, \mathcal{B}_{2}\right)$ the derived object of infinitesimal bimodule morphisms from $\mathcal{B}_{1}$ to $\mathcal{B}_{2}$. We also recall from [1, Section 5] the notation $\operatorname{sEmb}\left(-, \mathbb{R}^{d}\right)$ for the functor of standard embeddings.

Proposition 2.5 [1, Theorem 6.10; 15, Theorem 7.1] For $d>n$ and $k \leq \infty$, we have the weak equivalences

$$
T_{k} \overline{\operatorname{Emb}}_{c}\left(\mathbb{R}^{n}, \mathbb{R}^{d}\right) \simeq \operatorname{hInfBim}_{B_{n}}^{\leq k}\left(\operatorname{sEmb}\left(-, \mathbb{R}^{n}\right), B_{d}\right) \simeq \operatorname{hInfBim}_{B_{n}}^{\leq k}\left(B_{n}, B_{d}\right) .
$$

Notice that a version of Proposition 2.5 was proved [2] by Boavida de Brito and Weiss (they develop the details of the proof of that proposition). Notice also that Proposition 2.5 admits an algebraic version obtained by considering the functor

$$
C_{*} \overline{\operatorname{Emb}}_{c}\left(-, \mathbb{R}^{d}\right): \widetilde{\mathcal{O}}\left(\mathbb{R}^{n}\right) \longrightarrow \mathrm{Ch}_{*},
$$

in which $C_{*}(-)$ means the normalized singular chain complex functor.

Proposition 2.6 [1, Proposition 6.13] For $d>n$ and $k \leq \infty$ there are weak equivalences

$$
\begin{aligned}
T_{k} C_{*} \overline{\operatorname{Emb}}_{c}\left(\mathbb{R}^{n}, \mathbb{R}^{d}\right) & \simeq \operatorname{hInfBim}_{C_{*} B_{n}}^{\leq k}\left(C_{*} \operatorname{sEmb}\left(-, \mathbb{R}^{n}\right), C_{*} B_{d}\right) \\
& \simeq \operatorname{hInfBim}_{C_{*} B_{n}}^{\leq k}\left(C_{*} B_{n}, C_{*} B_{d}\right) .
\end{aligned}
$$

One can express (6) in terms of morphisms between infinitesimal bimodules over the commutative operad Com $=H_{0}\left(B_{d}\right)$. More precisely, one has Proposition 2.7, in which $C_{*}\left(S^{n-}\right)$ is viewed as an infinitesimal bimodule over Com, as follows. First of all, $S^{n}$ is the $n$-dimensional sphere viewed as the one-point compactification of $\mathbb{R}^{n}$, that is, $S^{n}=\mathbb{R}^{n} \cup\{\infty\}$ with $\infty$ as the base point. Therefore, by the second part of Example 3.2 and by Lemma 3.5 it follows that

$$
C_{*}\left(S^{n-}\right)=\left\{C_{*}\left(S^{n k}\right)\right\}_{k \geq 0}=\left\{C_{*}(\underbrace{S^{n} \times \cdots \times S^{n}}_{k})\right\}_{k \geq 0}
$$

is an infinitesimal bimodule over Com. Also, by the first part of Example 3.2, and by Lemma 3.5, the operad $H_{*}\left(B_{d}\right)$ is an infinitesimal bimodule over Com.

In the following proposition, the first weak equivalence (which essentially comes from the relative formality theorem [6, Theorem 1.4] of the inclusion of operads $B_{n} \hookrightarrow B_{d}$ ) is proved in [1, Proposition 7.1], and the second one in [1, Proposition 8.3]. Notice that Proposition 2.7 was first proved by Arone and Turchin [1] for $d \geq 2 n+1$. Actually the codimension condition $d \geq 2 n+1$, coming from the relative formality theorem of 
Lambrechts and Volić, was recently improved by Turchin and Willwacher (see [13]). They show, by a more careful analysis of the Lambrechts-Volić construction, that the relative formality theorem also holds for $d \geq n+2$.

Proposition 2.7 [1, Proposition 7.1 and Proposition 8.3] For $d \geq n+2$ and $k \leq \infty$, we have the weak equivalences

$$
\begin{aligned}
& T_{k} C_{*} \overline{\operatorname{Emb}}_{c}\left(\mathbb{R}^{n}, \mathbb{R}^{d}\right) \simeq \operatorname{hInfBim}_{C_{*} B_{n}}^{\leq k}\left(C_{*} B_{n}, H_{*}\left(B_{d} ; \mathbb{Q}\right),\right. \\
& T_{k} C_{*} \overline{\operatorname{Emb}}_{c}\left(\mathbb{R}^{n}, \mathbb{R}^{d}\right) \simeq \operatorname{hInfBim}_{\overline{\mathrm{Com}}}^{\leq k}\left(C_{*}\left(S^{n-}\right), H_{*}\left(B_{d} ; \mathbb{Q}\right) .\right.
\end{aligned}
$$

\subsection{The Taylor tower associated to $\overline{\operatorname{Emb}}_{c}\left(M, \mathbb{R}^{d}\right)$}

The goal here is to show that similar results as those mentioned in Section 2.1 hold for the space $\overline{\operatorname{Emb}}_{c}\left(M, \mathbb{R}^{d}\right)$, where $M$ is the manifold from Definition 2.1. We will prove them in a more general context. That is, instead of looking at $\overline{\operatorname{Emb}}_{c}\left(M, \mathbb{R}^{d}\right)$, we will look at the space $\overline{\operatorname{Emb}}_{c}\left(N, \mathbb{R}^{d}\right)$ in which $N$ denotes the complement of a compact subset of $\mathbb{R}^{n}$. Further, in Section 3, we will apply (in order to prove Theorem 1.1) the results of this section by taking $N=M$.

Let $K \subseteq[-1,1]^{n}$ be a compact subset with a finite number (greater than or equal to two) of connected components. Define $N$ to be the complement of $K$ in $\mathbb{R}^{n}$. Define also $\overline{\operatorname{Emb}}_{c}\left(N, \mathbb{R}^{d}\right)$ in the same way as the space $\overline{\operatorname{Emb}}_{c}\left(M, \mathbb{R}^{d}\right)$ from Definition 2.2. Here, the fixed immersion is a linear embedding

$$
\eta: \mathbb{R}^{n} \hookrightarrow \mathbb{R}^{d}, \quad \text { with } \eta\left(x_{1}, \ldots, x_{n}\right)=\left(0, \ldots, 0, x_{1}, \ldots, x_{n}\right) .
$$

Let $\mathcal{O}(N)$ be the category whose objects are open subsets of $N$ and morphisms are inclusions. In the general theory of Goodwillie and Weiss, to study the space $\operatorname{Emb}\left(N, \mathbb{R}^{d}\right)$ of embeddings of $N$ inside $\mathbb{R}^{d}$ we usually use the category $\mathcal{O}(N)$ as the source category for the functor $\operatorname{Emb}\left(-, \mathbb{R}^{d}\right)$. Here we want to study the space $\overline{\operatorname{Emb}}_{c}\left(N, \mathbb{R}^{d}\right)$ of compactly supported embeddings (modulo immersions) of $N$ in $\mathbb{R}^{d}$. So we need to define a suitable source category, and a suitable filtration of it.

Definition 2.8 - Define $\widetilde{\mathcal{O}}(N) \subseteq \mathcal{O}(N)$ to be the subcategory of open subsets whose complement in $N$ is bounded.

- Define $\widetilde{\mathcal{O}}_{k}(N)$ to be the subcategory of $\widetilde{\mathcal{O}}(N)$ consisting of subsets $U=V \cup W$ such that
(a) $V \cap W=\varnothing$,
(b) $V$ is the complement in $\mathbb{R}^{n}$ of a closed ball containing $K$ in $\mathbb{R}^{n}$,
(c) $W$ is the disjoint union of at most $k$ open balls. 
Recalling that we have fixed a linear embedding $\eta: \mathbb{R}^{n} \hookrightarrow \mathbb{R}^{d}$, one may define the contravariant functors

$$
\overline{\operatorname{Emb}}_{c}\left(-, \mathbb{R}^{d}\right): \widetilde{\mathcal{O}}(N) \longrightarrow \text { Top } \quad \text { and } \quad C_{*} \overline{\operatorname{Emb}}_{c}\left(-, \mathbb{R}^{d}\right): \widetilde{\mathcal{O}}(N) \longrightarrow \mathrm{Ch}_{*} .
$$

Taking these functors as inputs in Goodwillie-Weiss embedding calculus, we have the following two propositions, which are proved in a similar way as Proposition 2.5 and Proposition 2.6, respectively.

Proposition 2.9 For $d>n$ and $k \leq \infty$ there is a weak equivalence

$$
T_{k} \overline{\operatorname{Emb}}_{c}\left(N, \mathbb{R}^{d}\right) \simeq \operatorname{hInfBim}_{B_{n}}^{\leq k}\left(\operatorname{sEmb}(-, N), B_{d}\right) .
$$

Proposition 2.10 For $d>n$ and $k \leq \infty$ there is a weak equivalence

$$
T_{k} C_{*} \overline{\operatorname{Emb}}_{c}\left(N, \mathbb{R}^{d}\right) \simeq \operatorname{hInfBim}_{C_{*} B_{n}}^{\leq k}\left(C_{*} \operatorname{sEmb}(-, N), C_{*} B_{d}\right) .
$$

Applying now the relative formality theorem, which says that for $d \geq n+2$ the inclusion $B_{n} \hookrightarrow B_{d}$ is $\mathbb{R}$-formal (see [6, Theorem 1.4] and [13, Theorem 1]), we obtain the following proposition.

Proposition 2.11 For $d \geq n+2$ and $k \leq \infty$, there is a weak equivalence

$$
T_{k} C_{*}\left(\overline{\operatorname{Emb}}_{c}\left(N, \mathbb{R}^{d}\right) ; \mathbb{Q}\right) \simeq \operatorname{hInfBim}_{C_{*} B_{n}}^{\leq k}\left(C_{*}(\operatorname{sEmb}(-, N) ; \mathbb{Q}), H_{*}\left(B_{d} ; \mathbb{Q}\right)\right) .
$$

Proof The proof is the same as that of the second assertion of [1, Proposition 7.1].

We end this section with a proposition, which will be a key ingredient in the proof of Theorem 1.1. Before stating that proposition we fix some notation. Let $\hat{N}$ be the one-point compactification of $N$. That is,

$$
\widehat{N}=N \cup\{\infty\} .
$$

By $N \cup\{\infty\}$ we mean $g(N) \cup\{(0, \ldots, 0,1)\}$, where $g: \mathbb{R}^{n} \longrightarrow S^{n}$ is the inverse of the stereographic projection. The space $\widehat{N}$ is a pointed topological space with $\infty$ as the base point. As the case of $C_{*}\left(S^{n-}\right)$ from (7), we have a structure of an infinitesimal bimodule over Com on $C_{*}\left(\hat{N}^{\times-}\right)$.

Proposition 2.12 For $d \geq n+2$ and $k \leq \infty$, there is a weak equivalence

$$
T_{k} C_{*}\left(\overline{\operatorname{Emb}}_{c}\left(N, \mathbb{R}^{d}\right) ; \mathbb{Q}\right) \simeq \operatorname{hInfBim}_{\mathrm{Com}}^{\leq k}\left(C_{*}\left(\widehat{N}^{\times-}\right), H_{*}\left(B_{d} ; \mathbb{Q}\right)\right) .
$$


Proof The proof is the same as that of [1, Proposition 8.3], except that here we will work with the categories $\widetilde{\mathcal{O}}(N)$ and $\widetilde{\mathcal{O}}_{k}(N)$ instead of the categories $\widetilde{\mathcal{O}}\left(\mathbb{R}^{n}\right)$ and $\widetilde{\mathcal{O}}_{k}\left(\mathbb{R}^{n}\right)$. Recall that the two latter categories were defined at the beginning of Section 2.1.

Let $g: B_{n} \longrightarrow$ Com $=\{*\}_{p \geq 0}$ be the unique morphism of operads from $B_{n}$ to the topological commutative operad Com, and let $C_{*}(g): C_{*}\left(B_{n}\right) \longrightarrow C_{*}(\mathrm{Com})=\mathrm{Com}$ be the chain complex of $g$. The morphisms $g$ and $C_{*}(g)$ induce respectively pairs ind: $\operatorname{hInfBim}_{B_{n}} \rightleftarrows \operatorname{hInfBim}_{\mathrm{Com}}$ :res

and

$$
\text { ind: } \operatorname{hInfBim}_{C_{*} B_{n}} \rightleftarrows \operatorname{hInfBim}_{\mathrm{Com}} \text { :res }
$$

of adjoint functors. Here res is the restriction functor, and $\widetilde{\text { ind }}$ is the induction functor defined as follows. Let $\widetilde{\Gamma}\left(B_{n}\right)$ be the category whose object is a finite pointed set $(S, *)$, which is viewed as $|S|$ standard balls together with one standard antiball (an antiball is defined to be the complement of a ball). Morphisms in $\widetilde{\Gamma}\left(B_{n}\right)$ are standard embeddings, $\operatorname{sEmb}((S, *),(T, *))$, preserving the base point (the antiball playing the role of the base point). The category $\widetilde{\Gamma}\left(B_{n}\right)$ is filtered by the categories $\widetilde{\Gamma}_{\leq k}\left(B_{n}\right)$, where objects in $\widetilde{\Gamma}_{\leq k}\left(B_{n}\right)$ consist of one antiball together with (at most) $k$ open balls. It is proved (see [1, Proposition 4.9] for a more general statement) that the category of infinitesimal bimodules over $B_{n}$ is equivalent to the category of contravariant functors from $\widetilde{\Gamma}\left(B_{n}\right)$ to Top. We will thus identify these two categories. Let $X: \widetilde{\Gamma}\left(B_{n}\right) \longrightarrow$ Top be an infinitesimal bimodule over $B_{n}$. The object $\widetilde{\text { ind }}(X)$ is defined to be the homotopy left Kan extension of $X$ along $\widetilde{\Gamma}(g)$

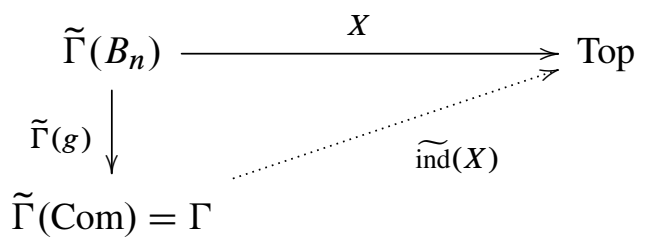

where $\Gamma$ is the category from Definition 3.1. Similarly, one can define the functor $\widetilde{\text { ind }}$ of (9). By noticing that $\operatorname{res}\left(H_{*}\left(B_{d}\right)\right)=H_{*}\left(B_{d}\right)$, and by using the adjunction (9), we deduce (from Proposition 2.11) that

$$
T_{k} C_{*}\left(\overline{\operatorname{Emb}}_{c}\left(N, \mathbb{R}^{d}\right) ; \mathbb{Q}\right) \simeq \operatorname{hInfBim}_{\operatorname{Com}}^{\leq k}\left(\widetilde{\operatorname{ind}}\left(C_{*}(\operatorname{sEmb}(-, N) ; \mathbb{Q})\right), H_{*}\left(B_{d} ; \mathbb{Q}\right)\right) .
$$

To end the proof it suffices to show that the functors $C_{*}\left(\widehat{N}^{\times-}\right)$and $\widetilde{\operatorname{ind}}\left(C_{*} \operatorname{sEmb}(-, N)\right)$ are weakly equivalent as infinitesimal bimodules over $\operatorname{Com}$. Since ind $\left(C_{*} \operatorname{sEmb}(-, N)\right)$ is the homotopy colimit of a certain diagram, and since the singular chain functor 
$C_{*}(-)$ commutes with homotopy colimits, it suffices to prove that there is a weak equivalence

$$
\widetilde{\operatorname{ind}}(\operatorname{sEmb}(-, N)) \simeq \widehat{N}^{\times-},
$$

holding in the category of contravariant functors from $\Gamma_{\leq k}$ to Top (here $\Gamma_{\leq k} \subseteq \Gamma$ is the subcategory whose objects are pointed sets $(S, *)$ with $|S| \leq k)$. The rest of the proof is devoted to (10). Notice first that

$$
\widetilde{\text { ind }} \operatorname{sEmb}(-, N) \simeq \underset{V \in \widetilde{\mathcal{O}}_{k}(N)}{\widetilde{\text { ind }}} \underset{\operatorname{hocolim}}{\operatorname{sEmb}}(-, V),
$$

and

$$
\underset{V \in \widetilde{\mathcal{O}}_{k}(N)}{\widetilde{\text { ind }}} \underset{\operatorname{hocolim}}{\operatorname{sEmb}}(-, V) \simeq \underset{V \in \widetilde{\mathcal{O}}_{k}(N)}{\operatorname{hocolim}} \underset{\text { ind }}{\operatorname{sEmb}}(-, V)
$$

Let $U \in \widetilde{\Gamma}_{\leq k}\left(B_{n}\right)$. Then, since $\operatorname{sEmb}(-, U): \widetilde{\Gamma}_{\leq k}\left(B_{n}\right) \longrightarrow$ Top is the free functor generated by $U$, it follows that $\widetilde{\pi n d} \operatorname{sEmb}(-, U)$ is the free functor generated by $\widetilde{\Gamma}(g)(U)$. That is,

$$
\widetilde{\text { ind }} \operatorname{sEmb}(-, U) \simeq \operatorname{Map}_{*}(-, \widetilde{\Gamma}(g)(U)): \Gamma_{\leq k} \longrightarrow \text { Top, }
$$

which is natural in $U$. Notice that $\operatorname{Map}_{*}(-, \widetilde{\Gamma}(g)(U))$ is not weakly equivalent to the functor $\operatorname{Map}_{*}(-, U)$ because the antiball of $U$ is not contractible. To correct this, let us define $\widehat{U}$ to be the one-point compactification of $U$, that is, $\widehat{U}=U \cup\{\infty\}$. Here the point $\infty$ is of course added to the antiball of $U$, and it is the base point of $\hat{U}$. We now have the weak equivalence

$$
\operatorname{Map}_{*}(-, \widetilde{\Gamma}(g)(U)) \simeq \operatorname{Map}_{*}(-, \widehat{U}),
$$

which is also natural in $U$. Combining (11)-(14), one has

$$
\widetilde{\text { ind } \operatorname{sEmb}}(-, N) \simeq \underset{V \in \widetilde{\mathcal{O}}_{k}(N)}{\operatorname{hocolim}} \operatorname{Map}_{*}(-, \hat{V}) .
$$

Since the right-hand side of (15) is weakly equivalent to $\operatorname{Map}_{*}(-, \widehat{N})=\widehat{N}^{\times-}$, the desired result follows.

\section{A cosimplicial model for the singular chain complex of the space of long links}

The goal of this section is to prove Theorem 1.1 announced in the introduction. Before doing that, we state some intermediate results. As in Section 2, the ground field in this section is $\mathbb{Q}$. 
Let us start with the definition of a right $\Gamma$-module.

Definition 3.1 - We define $\Gamma$ to be the category of finite pointed sets, the morphisms being the maps preserving the base point.

- A right $\Gamma-$ module is a contravariant functor from $\Gamma$ to chain complexes $\mathrm{Ch}_{*}$.

We denote by $R \bmod \Gamma$ the category of right $\Gamma$-modules. If $\mathcal{M}_{1}$ and $\mathcal{M}_{2}$ are two right $\Gamma$-modules, by $\mathrm{hRmod}_{\Gamma}\left(\mathcal{M}_{1}, \mathcal{M}_{2}\right)$, we denote the derived chain complex of right $\Gamma$-module morphisms from $\mathcal{M}_{1}$ to $\mathcal{M}_{2}$. Here are two examples of right $\Gamma$-modules that we look at in this paper.

Example 3.2 (i) The homology $H_{*}\left(B_{d}\right): \Gamma \longrightarrow \mathrm{Ch}_{*}$ defined by the formula

$$
H_{*}\left(B_{d}\right)\left(k_{+}\right)=H_{*}\left(B_{d}(k)\right),
$$

where $k_{+}$is a finite pointed set of cardinality $k+1$, is a right $\Gamma-$ module.

(ii) Let $X$ be a pointed topological space. The functor $C_{*}\left(X^{\times-}\right): \Gamma \longrightarrow \mathrm{Ch}_{*}$ defined by

$$
C_{*}\left(X^{\times-}\right)\left(k_{+}\right)=C_{*}\left(\operatorname{hom}_{\Gamma}\left(k_{+}, X\right)\right) \cong C_{*}\left(X^{\times k}\right)
$$

is a right $\Gamma$-module.

We are now going to define the cosimplicial chain complex $L_{*}^{\bullet}$ which appears in Theorem 1.1. From now on, if $X_{\bullet}$ is a simplicial set, we will denote by $X$ its geometric realization. Let $\left(\bigvee_{i=1}^{m} S^{1}\right)$. denote the simplicial model of the wedge $\bigvee_{i=1}^{m} S^{1}$ of $m$ copies of the circle, which has a unique 0 -simplex and exactly $m$ non-degenerate 1-simplices (see the beginning of Section 4 for the construction of that model). This simplicial model is actually a simplicial object in $\Gamma$, where the base points are taken to be the 0 -simplex and its degeneracies. Hence, we have the functor $\left(\bigvee_{i=1}^{m} S^{1}\right)_{\bullet}: \Delta^{\mathrm{op}} \longrightarrow \Gamma$, and we can therefore form the composite $H_{*}\left(B_{d}\right)\left(\left(\bigvee_{i=1}^{m} S^{1}\right)_{\bullet}\right): \Delta \longrightarrow \mathrm{Ch}_{*}$, which yields a cosimplicial chain complex.

Definition 3.3 The cosimplicial chain complex $L_{*}^{\bullet}$ is defined to be the composite

$$
L_{*}^{\bullet}=H_{*}\left(B_{d}\right)\left(\left(\bigvee_{i=1}^{m} S^{1}\right)_{\bullet}\right) .
$$

The following proposition is known to specialists, but its proof is written nowhere, to my knowledge. 
Proposition 3.4 Let $X_{\bullet}: \Delta \longrightarrow \Gamma$ be a simplicial object in the category $\Gamma$. Let $A: \Gamma \longrightarrow \mathrm{Ch}_{*}$ be a right $\Gamma$-module. Then there is a weak equivalence of chain complexes

$$
\operatorname{Tot} A\left(X_{\bullet}\right) \simeq \operatorname{hRmod}_{\Gamma}\left(C_{*}\left(\left|X_{\bullet}\right|^{\times-}\right), A\right)
$$

Proof We will work with a field $\mathbb{K}$ of characteristic 0 . For a set $S$ we denote by $\mathbb{K}[S]$ the vector space generated by $S$, which will be viewed as a chain complex concentrated in degree 0 .

We begin the proof by showing that there is an isomorphism

$$
C_{*}\left(\left|X_{\bullet}^{\times-}\right|\right) \cong C_{*}\left(\left|X_{\bullet}\right|^{\times-}\right)
$$

of right $\Gamma$-modules. To do that, let us consider the pair of functors

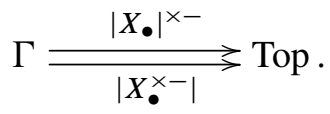

It is well known [7, Theorem 14.3] (since the simplicial set $X$ • is countable) that there is an isomorphism

$$
\left|X_{\bullet}\right| \times\left|X_{\bullet}\right| \stackrel{\cong}{\cong}\left|X_{\bullet} \times X_{\bullet}\right|
$$

in the category of topological spaces, and we can easily see that this isomorphism induces, for each $p_{+} \in \Gamma$, an isomorphism

$$
\phi_{p_{+}}:\left|X_{\bullet}\right|^{\times p} \cong\left|X_{\bullet}^{\times p}\right|
$$

which is natural in $p_{+}$. We thus get a natural isomorphism $\phi:\left|X_{\bullet}\right|^{\times-} \cong\left|X_{\bullet}^{\times-}\right|$ and, therefore, the isomorphism (17) holds in the category of right $\Gamma$-modules. From the latter isomorphism, we deduce the following one:

$$
\operatorname{hRmod}_{\Gamma}\left(C_{*}\left(\left|X_{\bullet}\right|^{\times-}\right), A\right) \cong \operatorname{hRmod}_{\Gamma}\left(C_{*}\left(\left|X_{\bullet}^{\times-}\right|\right), A\right) .
$$

In the second part of this proof, we are going to show, since the totalization $\operatorname{Tot}\left(A\left(X_{\bullet}\right)\right)$ is weakly equivalent to the homotopy limit of the $\Delta$-diagram $A\left(X_{\bullet}\right)$ in chain complexes, that the right-hand side of (18) is quasi-isomorphic to the homotopy limit of a certain $\Delta$-diagram. For the rest of this proof, the standard simplicial set $\Delta^{p}$. will be viewed as a simplicial object in $\Gamma$, where the base point of $\Delta_{k}^{p}=\operatorname{hom}_{\Delta}([k],[p])$ is taken to be the null morphism. We denote by $s \Gamma$ the category of simplicial objects in $\Gamma$, and by $N$ the Dold-Kan normalization functor. Let us consider the pair of contravariant functors

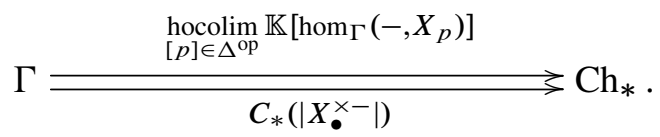


We want to build a natural weak equivalence between these two functors. So let $r_{+} \in \Gamma$ be a finite pointed set. Then the simplicial structure of $X$. induces a simplicial structure on $\operatorname{hom}_{\Gamma}\left(r_{+}, X_{\bullet}\right)$. By Yoneda's lemma, we have for each $p \geq 0$ the isomorphism

$\operatorname{hom}_{s \Gamma}\left(\Delta_{\bullet}^{p}, \operatorname{hom}_{\Gamma}\left(r_{+}, X_{\bullet}\right)\right)=\operatorname{hom}_{s \Gamma}\left(\operatorname{hom}_{\Delta}(\bullet,[p]), \operatorname{hom}_{\Gamma}\left(r_{+}, X_{\bullet}\right) \cong \operatorname{hom}_{\Gamma}\left(r_{+}, X_{p}\right)\right.$,

which implies that

$$
\begin{aligned}
\underset{[p] \in \Delta^{\mathrm{op}}}{\operatorname{hocolim}} \mathbb{K}\left[\operatorname{hom}_{\Gamma}\left(r_{+}, X_{p}\right)\right] & \cong \underset{[p] \in \Delta^{\mathrm{op}}}{\operatorname{hocolim}} \mathbb{K}\left[\operatorname{hom}_{s}\left(\Delta_{\bullet}^{p}, \operatorname{hom}_{\Gamma}\left(r_{+}, X_{\bullet}\right)\right)\right] \\
& \simeq N V_{\bullet}\left(r_{+}\right) .
\end{aligned}
$$

Here $V_{\bullet}\left(r_{+}\right)$is the simplicial chain complex defined by the formula

$$
V_{p}\left(r_{+}\right)=\mathbb{K}\left[\operatorname{hom}_{s \Gamma}\left(\Delta_{\bullet}^{p}, \operatorname{hom}_{\Gamma}\left(r_{+}, X_{\bullet}\right)\right)\right]
$$

Notice that the isomorphism (20) and the weak equivalence (21) are natural in $r_{+}$. On the other hand, let $W_{\bullet}\left(r_{+}\right)$be the simplicial chain complex defined by

$$
W_{p}\left(r_{+}\right)=\mathbb{K}\left[\operatorname{hom}_{\mathrm{Top}}\left(\Delta^{p},\left|X_{\bullet}^{\times r}\right|\right)\right] .
$$

Then the associated chain complex is nothing other than the singular chain complex $C_{*}\left(\left|X_{\bullet}^{\times_{r}}\right|\right)$. Therefore, since the chain complex associated to a simplicial abelian group is quasi-isomorphic to its Dold-Kan normalization, there is a natural quasi-isomorphism

$$
C_{*}\left(\left|X_{\bullet}^{\times r}\right|\right) \simeq W_{\bullet}\left(r_{+}\right)
$$

We have just defined a pair of contravariant functors

$$
\Gamma \stackrel{N V_{\bullet}}{\underset{N W_{\bullet}}{\stackrel{\longrightarrow}{\rightleftharpoons}}} \mathrm{Ch}_{*}
$$

Define now

$$
\alpha_{r_{+}}: N V_{p}\left(r_{+}\right) \longrightarrow N W_{p}\left(r_{+}\right) \text {by } \alpha_{r_{+}}(f)=|f|,
$$

where $f: \Delta_{\bullet}^{p} \longrightarrow \operatorname{hom}_{\Gamma}\left(r_{+}, X_{\bullet}\right)$ is a morphism in simplicial sets. It is straightforward to check that $\alpha: N V_{\bullet} \longrightarrow N W_{\bullet}$ is a quasi-isomorphism natural in $r_{+}$. This implies (with (20)-(22)) that there is a quasi-isomorphism

$$
\underset{[p] \in \Delta^{\mathrm{op}}}{\operatorname{hocolim}} \mathbb{K}\left[\operatorname{hom}_{\Gamma}\left(-, X_{p}\right)\right] \simeq C_{*}\left(\left|X_{\bullet}^{\times-}\right|\right) .
$$


in the category of right $\Gamma$-modules. We end the proof with the following summary:

$$
\begin{aligned}
\operatorname{hRmod}_{\Gamma}\left(C_{*}\left(\left|X_{\bullet}\right|^{\times-}\right), A\right) & \cong \operatorname{hRmod}_{\Gamma}\left(C_{*}\left(\left|X_{\bullet}^{\times-}\right|\right), A\right) \text { by }(18) \\
& \simeq \operatorname{hRmod} \Gamma\left(\underset{[p] \in \Delta^{\mathrm{op}}}{\operatorname{hocolim}} \mathbb{K}\left[\operatorname{hom}_{\Gamma}\left(-, X_{p}\right)\right], A\right) \\
& \simeq \operatorname{holim}_{[p] \in \Delta} \operatorname{hRmod} \Gamma\left(\mathbb{K}\left[\operatorname{hom}_{\Gamma}\left(-, X_{p}\right)\right], A\right) \\
& \cong \operatorname{holim}_{[p] \in \Delta}\left(A\left(X_{p}\right)\right) \quad \text { by Yoneda's lemma } \\
& \cong \operatorname{Tot}\left(A\left(X_{\bullet}\right)\right) .
\end{aligned}
$$

Before starting the proof of Theorem 1.1, we need to state Lemma 3.5 and Theorem 3.6. The first one is an immediate consequence of [1, Propostion 4.9].

Lemma 3.5 The category of infinitesimal bimodules over the commutative operad is equivalent to the category of right $\Gamma$-modules. That is,

$$
\operatorname{InfBim}_{\mathrm{Com}} \cong \operatorname{Rmod}_{\Gamma} .
$$

Recalling the definition of $M_{n}$ from (3), one has the following result, which is proved using Goodwillie-Weiss techniques for embedding calculus [17; 3].

Theorem 3.6 For $d \geq 2 n+2$, there is a weak equivalence

$$
C_{*}\left(\overline{\operatorname{Emb}}_{c}\left(M_{n}, \mathbb{R}^{d}\right) ; \mathbb{Q}\right) \simeq T_{\infty} C_{*}\left(\overline{\operatorname{Emb}}_{c}\left(M_{n}, \mathbb{R}^{d}\right) ; \mathbb{Q}\right) .
$$

Proof There is a weak equivalence

$$
C_{*}\left(\overline{\operatorname{Emb}}_{c}\left(M_{n}, \mathbb{R}^{d}\right) ; \mathbb{Q}\right) \simeq T_{\infty} C_{*}\left(\overline{\operatorname{Emb}}_{c}\left(M_{n}, \mathbb{R}^{d}\right) ; \mathbb{Q}\right)
$$

for $d \geq 2 \operatorname{dim}\left(M_{n}\right)+2=2 n+4$. Since the important quantity regarding the source manifold is not the actual dimension, but the homotopy dimension, it follows that (24) also holds for $d \geq 2 n+2$ (notice that the manifold $M_{n}$ is essentially an $(m+1)$ punctured euclidean space $\mathbb{R}^{n+1}$, and therefore its homotopy dimension is $n$ ). This is the relative (or boundary) stronger formulation of the excision estimates of Goodwillie and Klein.

The following remark is straightforward.

Remark 3.7 The one-point compactification of $M$, denoted $\widehat{M}=M \cup\{\infty\}$ (for the meaning of "one-point compactification" see the paragraph just before Proposition 2.12), is weakly equivalent to the wedge of $m$ copies of the circle. That is,

$$
\widehat{M} \simeq \bigvee_{i=1}^{m} S^{1} .
$$


We are now ready to prove Theorem 1.1 , which states that the cosimplicial chain complex $L_{*}^{\bullet}$ defined above (see Definition 3.3) is a cosimplicial model for the singular chain complex of the space $\overline{\mathcal{L}}_{m}^{d}$ of long links of $m$ strands in $\mathbb{R}^{d}$.

Proof of Theorem 1.1 For $d \geq 4$, we have the following weak equivalences:

$$
\begin{aligned}
C_{*}\left(\overline{\mathcal{L}}_{m}^{d}\right) \otimes \mathbb{Q} & \simeq C_{*}\left(\overline{\operatorname{Emb}}_{c}\left(M, \mathbb{R}^{d}\right) ; \mathbb{Q}\right) & & (\text { Proposition 2.3) } \\
& \simeq T_{\infty} C_{*}\left(\overline{\operatorname{Emb}}_{c}\left(M, \mathbb{R}^{d}\right) ; \mathbb{Q}\right) & & (\text { Theorem 3.6 } \\
& \simeq \operatorname{hInfBim}_{C_{*} B_{2}}\left(C_{*} \operatorname{sEmb}(-, M), C_{*} B_{d}\right) & & (\text { Proposition } 2.10 \\
& \simeq \operatorname{hInfBim}_{C_{*} B_{2}}\left(C_{*} \operatorname{sEmb}(-, M), H_{*} B_{d}\right) & & \text { with } N=M) \\
& & & \text { (Proposition 2.11 } \\
& \simeq \operatorname{hInfBim}_{\operatorname{Com}}\left(C_{*}\left(\hat{M}^{\times-}\right), H_{*} B_{d}\right) & & (\text { Proposition 2.12 } \\
& \simeq \operatorname{hRmod}_{\Gamma}\left(C_{*}\left(\hat{M}^{\times-}\right), H_{*} B_{d}\right) & & \text { with } N=M) \\
& \simeq \operatorname{hRmod}_{\Gamma}\left(C_{*}\left(\left(\bigvee_{i=1}^{m} S^{1}\right)^{\times-}\right), H_{*} B_{d}\right) & & \text { (25) } \\
& \simeq \operatorname{Tot}_{*} B_{d}\left(\left(\bigvee_{i=1}^{m} S^{1}\right) .\right) & & \text { (Proposition 3.4) } \\
& =\operatorname{Tot}_{*}^{\bullet} & & \text { (Definition 3.3). }
\end{aligned}
$$

\section{Collapsing of the Bousfield-Kan spectral sequence associ- ated to the Munson-Volić cosimplicial model}

The goal of this section is to prove Theorem 1.4 stated in the introduction. We begin by giving the simplicial model of the wedge of $m$ copies of the circle. Next we state and prove the crucial Lemma 4.2. Finally we prove Theorem 1.4. As in the previous sections, the ground field here is $\mathbb{Q}$.

Let $\Delta_{\text {. }}^{1}$ denote the simplicial model of the standard 1 -simplex $\Delta^{1}$, and let $\partial \Delta^{1}$. denote its boundary. Recall that $\Delta_{p}^{1}$ is a nondecreasing sequence of length $p+1$ on the alphabet $\{0,1\}$. Define the simplicial set $S_{\bullet}^{1}$ to be the quotient

$$
S_{\bullet}^{1}=\frac{\Delta_{\bullet}^{1}}{\partial \Delta_{\bullet}^{1}} .
$$

It is clear that $S_{\bullet}^{1}$ is a simplicial model of the circle $S^{1}$. Notice that each $S_{p}^{1}$ is a finite set pointed at

$$
*=\underbrace{0 \cdots 0}_{p+1} \sim \underbrace{1 \cdots 1}_{p+1},
$$


and faces and degeneracies preserve the base point. Therefore $S_{\bullet}^{1}$ is a simplicial object in $\Gamma$. Define now the simplicial set $\left(\bigvee_{i=1}^{m} S^{1}\right)$. to be the wedge of $m$ copies of the simplicial set $S_{\bullet}^{1}$ :

$$
\left(\bigvee_{i=1}^{m} S^{1}\right)_{\bullet}=\bigvee_{i=1}^{m}\left(S_{\bullet}^{1}\right) .
$$

The following proposition is well known in the literature.

Proposition 4.1 The simplicial set $\left(\bigvee_{i=1}^{m} S^{1}\right)$. is a simplicial model for the wedge $\bigvee_{i=1}^{m} S^{1}$. Moreover, for each $p \geq 0$, the finite pointed set $\left(\bigvee_{i=1}^{m} S^{1}\right)_{p}$ is of cardinality $m p+1$. That is,

$$
\left|\left(\bigvee_{i=1}^{m} S^{1}\right)_{p}\right|=m p+1
$$

Proof It is straightforward to check that $\left(\bigvee_{i=1}^{m} S^{1}\right)$. is a simplicial model of $\bigvee_{i=1}^{m} S^{1}$. Let $p \geq 0$. Since $\left|S_{p}^{1}\right|=p+1$, by the definition of the wedge, it follows that $\left|\left(\bigvee_{i=1}^{m} S^{1}\right)_{p}\right|=m p+1$.

Recall now some notation about spectral sequences. For a cosimplicial chain complex $C_{*}^{\bullet}$, the associated total complex admits a natural filtration by the cosimplicial degree. We denote by $\left\{E^{r}\left(C_{*}^{\bullet}\right)\right\}_{r \geq 0}$ the spectral sequence induced by this filtration.

In the rest of this paper, we will denote by $\mathcal{K}_{d}^{m \bullet}$ the Munson-Volić cosimplicial model [9] for the space of long links of $m$ strands. Notice that $\mathcal{K}_{d}^{m \bullet}$ is built in the same spirit as Sinha's cosimplicial model [11] for the space of long knots.

Lemma 4.2 For $d \geq 3$, the $E^{1}$ pages of the spectral sequences $\left\{E^{r}\left(L_{*}^{\bullet}\right)\right\}_{r \geq 0}$ and $\left\{E^{r}\left(C_{*}\left(\mathcal{K}_{d}^{m \bullet} ; \mathbb{Q}\right)\right)\right\}_{r \geq 0}$ are isomorphic. That is,

$$
\left\{E^{r}\left(L_{*}^{\bullet}\right)\right\}_{r=1} \cong\left\{E^{r}\left(C_{*}\left(\mathcal{K}_{d}^{m \bullet} ; \mathbb{Q}\right)\right)\right\}_{r=1} .
$$

Before proving Lemma 4.2, we recall the Com-infinitesimal bimodule structures of $H_{*}\left(B_{d}\right)$ and $H_{*}\left(\mathcal{K}_{d}\right)$ (note that $\mathcal{K}_{d}$ is the Kontsevich operad, which was defined and studied in [11, Definition 4.1 and Theorem 4.5]). First, as in the previous sections, Com is the $0^{\text {th }}$ homology group of the little $d$-disks operad $B_{d}$. That is, Com $=H_{0}\left(B_{d}\right)$. Here $H_{0}(-)$ is viewed as a chain complex concentrated in degree 0 . Next we endow the homology $H_{*}\left(B_{d}\right)$ with the Com-infinitesimal bimodule structure induced by the obvious morphism Com $\longrightarrow H_{*}\left(B_{d}\right)$. The homology $H_{*}\left(\mathcal{K}_{d}\right)$ is endowed with a similar Com-infinitesimal bimodule structure, since [10, Theorem 5.10], which states that the operads $B_{d}$ and $\mathcal{K}_{d}$ are weakly equivalent, implies the existence of an isomorphism $H_{*}\left(B_{d}\right) \stackrel{\cong}{\rightrightarrows} H_{*}\left(\mathcal{K}_{d}\right)$. 
Proof of Lemma 4.2 Since the diagram

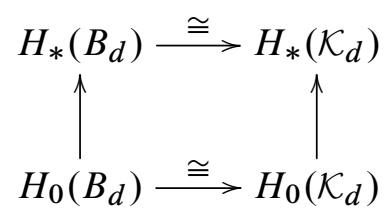

is commutative, it follows that the upper isomorphism holds in the category $\operatorname{InfBim}_{\mathrm{Com}}$. Therefore, since an infinitesimal bimodule over Com is the same thing as a right $\Gamma$-module (see Lemma 3.5), the same isomorphism $\left(H_{*}\left(B_{d}\right) \cong H_{*}\left(\mathcal{K}_{d}\right)\right)$ holds in the category of right $\Gamma$-modules. This implies that the isomorphism

$$
\begin{aligned}
L_{*}^{\bullet} & =H_{*}\left(B_{d}\right)\left(\left(\bigvee_{i=1}^{m} S^{1}\right)_{\bullet}\right) \\
& \cong H_{*}\left(\mathcal{K}_{d}\right)\left(\left(\bigvee_{i=1}^{m} S^{1}\right) \bullet\right) \\
& =H_{*}\left(\mathcal{K}_{d}^{m \bullet}\right) \quad(\text { Proposition } 4.1)
\end{aligned}
$$

holds in the category of cosimplicial chain complexes, thus completing the proof.

Lemma 4.3 For $d \geq 3$ the spectral sequence $\left\{E^{r}\left(L_{*}^{\bullet}\right)\right\}_{r \geq 0}$ collapses at the $E^{2}$ page rationally.

Proof By Proposition 4.1 and Definition 3.3, we have $L_{*}^{p}=H_{*}\left(B_{d}(m p)\right)$ for each $p \geq 0$. Since the homology $H_{*}\left(B_{d}(m p)\right.$ is a chain complex with 0 differential, it follows that the vertical differential in the bicomplex associated to $L_{*}^{\bullet}$ is trivial. Therefore, the spectral sequence $\left\{E^{r}\left(L_{*}^{\bullet}\right)\right\}_{r \geq 0}$ collapses at the $E^{2}$ page.

We are now ready to prove Theorem 1.4.

Proof of Theorem 1.4 The proof follows from the following three points:

- The $E^{1}$ pages of $\left\{E^{r}\left(L_{*}^{\bullet}\right)\right\}_{r \geq 0}$ and $\left\{E^{r}\left(C_{*}\left(\mathcal{K}_{d}^{m \bullet} ; \mathbb{Q}\right)\right)\right\}_{r \geq 0}$ are isomorphic by Lemma 4.2.

- For $d \geq 4$, the spectral sequences $\left\{E^{r}\left(L_{*}^{\bullet}\right)\right\}_{r \geq 0}$ and $\left\{E^{r}\left(C_{*}\left(\mathcal{K}_{d}^{m \bullet} ; \mathbb{Q}\right)\right)\right\}_{r \geq 0}$ have the same abutment by Theorem 1.1.

- The spectral sequence $\left\{E^{r}\left(L_{*}^{\bullet}\right)\right\}_{r \geq 0}$ collapses at the $E^{2}$ page by Lemma 4.3. 


\section{High-dimensional analogues of spaces of long links}

The goal of this short section is to show that our method enables us also to get the collapsing at the $E^{2}$ page of the spectral sequence computing the rational homology of the high-dimensional analogues of spaces of long links.

Let us start with a definition. Consider the space $\operatorname{Emb}_{c}\left(\bigsqcup_{i=1}^{m} \mathbb{R}^{n}, \mathbb{R}^{d}\right)$ of compactly supported embeddings of $\bigsqcup_{i=1}^{m} \mathbb{R}^{n}$ inside $\mathbb{R}^{d}$. Consider also $\operatorname{Imm}_{c}\left(\bigsqcup_{i=1}^{m} \mathbb{R}^{n}, \mathbb{R}^{d}\right)$.

Definition 5.1 The high-dimensional analogue of the space of long links is the homotopy fiber of the inclusion

$$
\operatorname{Emb}_{c}\left(\bigsqcup_{i=1}^{m} \mathbb{R}^{n}, \mathbb{R}^{d}\right) \hookrightarrow \operatorname{Imm}_{c}\left(\bigsqcup_{i=1}^{m} \mathbb{R}^{n}, \mathbb{R}^{d}\right),
$$

and it is denoted by $\overline{\operatorname{Emb}}_{c}\left(\bigsqcup_{i=1}^{m} \mathbb{R}^{n}, \mathbb{R}^{d}\right)$.

As in the case of long links, let us consider the cosimplicial chain complex

$$
L_{*}^{n \bullet}:=H_{*}\left(B_{d}, \mathbb{Q}\right)\left(\left(\bigvee_{i=1}^{m} S^{n}\right) \bullet\right)
$$

in which $\left(\bigvee_{i=1}^{m} S^{n}\right)$. is the simplicial model (built in the similar way as $\left(\bigvee_{i=1}^{m} S^{1}\right)$ • ) of the wedge $\bigvee_{i=1}^{m} S^{n}$ of $m$ copies of the $n$-dimensional sphere $S^{n}$. The following theorem gives a cosimplicial model for the singular chain complex $C_{*} \overline{\operatorname{Emb}}_{c}\left(\bigsqcup_{i=1}^{m} \mathbb{R}^{n}, \mathbb{R}^{d}\right)$.

Theorem 5.2 For $d \geq 2 n+2$ there is a weak equivalence

$$
\operatorname{Tot} L_{*}^{n \bullet} \simeq C_{*}\left(\overline{\operatorname{Emb}}_{c}\left(\bigsqcup_{i=1}^{m} \mathbb{R}^{n}, \mathbb{R}^{d}\right)\right) \otimes \mathbb{Q} .
$$

Proof The proof works exactly as that of Theorem 1.1 given in Section 3. It suffices to

- use (4) from Remark 2.4,

- replace $M$ by $M_{n}$ (recall that the open submanifold $M_{n} \subseteq \mathbb{R}^{n+1}$ was defined in (3)),

- replace $B_{2}$ by $B_{n+1}$, and of course $S^{1}$ by $S^{n}$ and $L_{*}^{\bullet}$ by $L_{*}^{n \bullet}$.

The rest remains unchanged.

The following corollary is a generalization of Corollary 1.2. It is also an immediate consequence of Theorem 5.2. 
Corollary 5.3 For $d \geq 2 n+2$ there is an isomorphism

$$
H_{*}\left(\overline{\operatorname{Emb}}_{c}\left(\bigsqcup_{i=1}^{m} \mathbb{R}^{n}, \mathbb{R}^{d}\right) ; \mathbb{Q}\right) \cong H H^{\vee_{i=1}^{m} S^{n}}\left(H_{*}\left(B_{d} ; \mathbb{Q}\right)\right) \text {. }
$$

Let us consider now the spectral sequence $\left\{E^{r}\left(L_{*}^{n \bullet}\right)\right\}_{r \geq 0}$, the Bousfield-Kan spectral sequence associated to $L_{*}^{n \bullet}$. It is clear by Theorem 5.2 that it converges to the homology $H_{*}\left(\overline{\operatorname{Emb}}_{c}\left(\bigsqcup_{i=1}^{m} \mathbb{R}^{n}, \mathbb{R}^{d}\right) ; \mathbb{Q}\right)$ when $d \geq 2 n+2$. We can prove exactly as Lemma 4.3 above that this spectral sequence collapses at the $E^{2}$ page, which gives the following result.

Proposition 5.4 For $d \geq 2 n+2$, the spectral sequence $\left\{E^{r}\left(L_{*}^{n \bullet}\right)\right\}_{r \geq 0}$ computing the rational homology $H_{*}\left(\overline{\mathrm{Emb}}_{c}\left(\bigsqcup_{i=1}^{m} \mathbb{R}^{n}, \mathbb{R}^{d}\right) ; \mathbb{Q}\right)$ collapses at the $E^{2}$ page rationally.

\section{Poincaré series for the space of long links modulo $m$ copies of long knots}

The aim of this section is to prove that the radius of convergence of the Poincare series for the pair formed by the space of long links and the space of $m$ copies of long knots tends to 0 as $m$ goes to the infinity. We also state a conjecture followed by a theorem concerning the radius of convergence for that pair. Here, the abbreviation $H^{*} \mathrm{BKSS}$ means cohomology Bousfield-Kan spectral sequence.

Let us start by defining expressions that appear in the title of the section.

Definition 6.1 Let $X$ be a topological space.

- For $k \geq 0$ the $k^{\text {th }}$ Betti number, $b_{k}(X)$, of $X$ is the rank of its $k^{\text {th }}$ homology group $H_{k}(X)$.

- The Poincaré series of $X$, denoted by $P_{X}[x]$, is the series

$$
P_{X}[x]=\sum_{k=0}^{\infty} b_{k}(X) x^{k} .
$$

Up to now we have denoted the space of long knots by $\overline{\operatorname{Emb}}_{c}\left(\mathbb{R}, \mathbb{R}^{d}\right)$. For the sake of simplicity, we will denote it here by $\mathcal{K}$. Let $\mathcal{K}^{\times m}$ denote the space of $m$ copies of long knots. Recall also the notation $\overline{\mathcal{L}}_{m}^{d}$ for the space of long links (modulo immersions) of $m$ strands in $\mathbb{R}^{d}$. It is clear that $\mathcal{K}^{\times m}$ is a subspace of $\overline{\mathcal{L}}_{m}^{d}$.

Definition 6.2 The pair $\left(\overline{\mathcal{L}}_{m}^{d}, \mathcal{K}^{\times m}\right)$ is called the space of long links modulo $m$ copies of long knots. 
In [4], Komawila and Lambrechts studied the Euler series of the $E_{1}$ page of the $H^{*}$ BKSS associated to the Munson-Volić cosimplicial model for the space of long links, and they obtained the following results. Recall that the Euler series associated to a bigraded vector space $V=\left\{V_{p, q}\right\}_{p, q \geq 0}$ is defined by

$$
\chi(V)[x]=\sum_{q=0}^{\infty}\left(\sum_{p=0}^{\infty}(-1)^{p} \operatorname{dim} V_{p, q}\right) x^{q} .
$$

Theorem 6.3 [4, Theorem 5.1] For $d \geq 4$ the Euler series $\chi\left(E_{1}\right)[x]$ of the $E_{1}$ page of the $H^{*} B K S S$ associated to $\mathcal{L}_{m}^{d \bullet}$ is given by

$$
\chi\left(E_{1}\right)[x]=\frac{1}{\left(1-x^{d-1}\right)\left(1-2 x^{d-1}\right) \cdots\left(1-m x^{d-1}\right)} .
$$

The following corollary gives the Euler series of the pair $\left(\overline{\mathcal{L}}_{m}^{d}, \mathcal{K}^{\times m}\right)$.

Corollary 6.4 [4] For $d \geq 4$ the Euler series of the $E_{2}$ page of the $H^{*} B K S S$ associated to the pair $\left(\mathcal{L}_{m}^{d \bullet},\left(\mathcal{K}_{d}^{\bullet}\right)^{\times m}\right)$ is given by

$$
\chi\left(E_{2}\right)[x]=\frac{1}{\left(1-x^{d-1}\right)\left(1-2 x^{d-1}\right) \cdots\left(1-m x^{d-1}\right)}-\frac{1}{\left(1-x^{d-1}\right)^{m}} .
$$

Proof Recall first that the pair $\left(\overline{\mathcal{L}}_{m}^{d}, \mathcal{K}^{\times m}\right)$ admits a cosimplicial model $\left(\mathcal{L}_{m}^{d \bullet},\left(\mathcal{K}_{d}^{\bullet}\right)^{\times m}\right)$. The second component of that cosimplicial model is just the product $\left(\mathcal{K}_{d}^{\bullet}\right)^{\times m}$ of $m$ copies of the Sinha cosimplicial model $\mathcal{K}_{d}^{\bullet}$. The proof of the corollary comes from Theorem 6.3 and the fact that the retraction (up to homotopy) $\overline{\mathcal{L}}_{m}^{d} \longrightarrow \mathcal{K}^{\times m}$ (see [4, Section 2.1] for an explicit definition of that retraction) holds at the level of cosimplicial models, so that we have the isomorphism

$$
\left\{E_{r}\left(\left(\overline{\mathcal{L}}_{m}^{d}, \mathcal{K}^{\times m}\right)\right)\right\}_{r \geq 0} \cong \frac{\left\{E_{r}\left(\overline{\mathcal{L}}_{m}^{d}\right)\right\}_{r \geq 0}}{\left\{E_{r}\left(\mathcal{K}^{\times m}\right)\right\}_{r \geq 0}}
$$

of spectral sequences.

From Corollary 6.4 and Theorem 1.4, we deduce the exponential growth of the Betti numbers of the pair $\left(\overline{\mathcal{L}}_{m}^{d}, \mathcal{K}^{\times m}\right)$.

Proposition 6.5 For $d \geq 4$ the Betti numbers of the pair $\left(\overline{\mathcal{L}}_{m}^{d}, \mathcal{K}^{\times m}\right)$ grow at least exponentially.

Proof By (28) and Theorem 1.4, the $H^{*}$ BKSS of the pair $\left(\mathcal{L}_{m}^{d \bullet},\left(\mathcal{K}_{d}^{\bullet}\right)^{\times m}\right)$ collapses at the $E^{2}$ page. Moreover, the coefficients of (27) grow exponentially at a rate of $m^{1 / d-1}>1$, and by [4, Proposition 4.5] the Betti numbers of the pair $\left(\overline{\mathcal{L}}_{m}^{d}, \mathcal{K}^{\times m}\right)$ have the same growth. 
One can also see Proposition 6.5 as a consequence of a theorem of Turchin [14, Theorem 17.1], which states that the Betti numbers of the space $\mathcal{K}$ of long knots grow at least exponentially. Notice first that the concatenation operation endows $\overline{\mathcal{L}}_{m}^{d}$ and $\mathcal{K}^{\times m}$ with the structure, denoted $\times$, of an $H$-space. Let $\mathbf{1} \in \mathcal{K}^{\times m}$ denote the unit, and consider the diagram

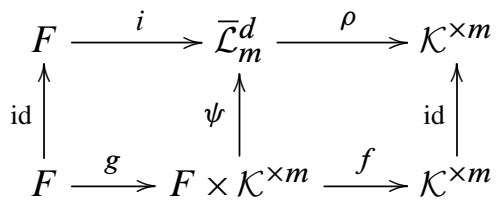

where

- $F$ is the fiber of $\rho$ over the unit $\mathbf{1}$, id is the identity map,

- the map $\psi$ is defined by $\psi(x, y)=i(x) \times s(y)$, where $s: \mathcal{K}^{\times m} \longrightarrow \overline{\mathcal{L}}_{m}^{d}$ is a section of $\rho$,

- the maps $g$ and $f$ are defined by $g(x)=(x, \mathbf{1})$ and $f(x, y)=y$,

- the map $\rho$ is the one constructed in [4, Section 2].

It is clear that the left square of (29) commutes. The right square also commutes, because

$$
\begin{aligned}
\rho(\psi(x, y)) & =\rho(i(x) \times s(y)) \\
& =\rho(i(x)) \times \rho(s(y)) \quad \text { (because } \rho \text { is a morphism of } H \text {-spaces) } \\
& =\mathbf{1} \times y \quad \text { (because } s \text { is a section of } \rho) \\
& =f(x, y) .
\end{aligned}
$$

This implies that the triple (id, $\psi$, id) is a morphism of fibrations, and therefore the space $\overline{\mathcal{L}}_{m}^{d}$ is homeomorphic to the product $F \times \mathcal{K}^{\times m}$. We thus have the inequality

$$
\operatorname{dim} H_{*}\left(\overline{\mathcal{L}}_{m}^{d}\right)>\operatorname{dim} H_{*}\left(\mathcal{K}^{\times m}\right) .
$$

Since $\operatorname{dim} H_{*}(\mathcal{K})$ grows at least exponentially [14, Theorem 17.1], it follows that $\operatorname{dim} H_{*}\left(\mathcal{K}^{\times m}\right)$ also grows at least exponentially, and the proposition follows.

Our proof has some consequences. We have seen that the Betti numbers of the pair $\left(\overline{\mathcal{L}}_{m}^{d}, \mathcal{K}^{\times m}\right)$ grow exponentially at a rate of $m^{1 /(d-1)}$. This implies the following corollary.

Corollary 6.6 For $d \geq 4$, the radius of convergence of the Poincare series for the pair $\left(\overline{\mathcal{L}}_{m}^{d}, \mathcal{K}^{\times m}\right)$ is less than or equal to $(1 / m)^{1 /(d-1)}$, and therefore tends to 0 as $m$ goes to $\infty$. 
Let us denote by $\mathrm{RC}(X)$ the radius of convergence of the Poincaré series for a space $X$. For the space of long knots we will denote it by $R$.

Remark 6.7 As a consequence of Theorem 1.8 we have,

$$
\operatorname{RC}\left(\overline{\mathcal{L}}_{m}^{d}, \mathcal{K}^{\times m}\right) \leq(1 / \sqrt{2})^{1 /(d-1)} .
$$

Our approach gives a better upper bound for this radius thanks to Corollary 6.6.

We end this section with a conjecture having a nice consequence.

Conjecture 6.8 The radius of convergence of the Poincaré series of the space of long knots (modulo immersions) is greater than 0 . That is, $R>0$.

Corollary 6.6 tells us that the radius of convergence of the Poincare series for $\left(\overline{\mathcal{L}}_{m}^{d}, \mathcal{K}^{\times m}\right)$ is less than or equal to $(1 / m)^{1 /(d-1)}$, but does not tell us that it is less than $R$. We therefore have the following theorem.

Theorem 6.9 If Conjecture 6.8 is true, then for $d \geq 4$ and for $m>1 / R^{d-1}$ the radius of convergence of the Poincaré series for the pair $\left(\overline{\mathcal{L}}_{m}^{d}, \mathcal{K}^{\times m}\right)$ is less than $R$. That is, $\operatorname{RC}\left(\overline{\mathcal{L}}_{m}^{d}, \mathcal{K}^{\times m}\right)<R$.

Proof The proof comes immediately from Corollary 6.6 and the hypothesis that $m>1 / R^{d-1}$.

\section{References}

[1] G Arone, V Turchin, On the rational homology of high-dimensional analogues of spaces of long knots, Geom. Topol. 18 (2014) 1261-1322 MR3228453

[2] P Boavida de Brito, M Weiss, Manifold calculus and homotopy sheaves, Homology Homotopy Appl. 15 (2013) 361-383 MR3138384

[3] T G Goodwillie, M Weiss, Embeddings from the point of view of immersion theory, II, Geom. Topol. 3 (1999) 103-118 MR1694808

[4] G Komawila, P Lambrechts, Euler series, Stirling numbers and the growth of the homology of the space of long links, Bull. Belg. Math. Soc. Simon Stevin 20 (2013) 843-857 MR3160593

[5] P Lambrechts, V Turchin, I Volić, The rational homology of spaces of long knots in codimension > 2, Geom. Topol. 14 (2010) 2151-2187 MR2740644

[6] P Lambrechts, I Volić, Formality of the little $N$-disks operad, Mem. Amer. Math. Soc. 1079, Amer. Math. Soc. (2014) MR3220287 
[7] J P May, Simplicial objects in algebraic topology, Van Nostrand Mathematical Studies 11, D. Van Nostrand, Princeton (1967) MR0222892

[8] S Moriya, Multiplicative formality of operads and Sinha's spectral sequence for long knots, Kyoto J. Math. 55 (2015) 17-27 MR3323525

[9] B A Munson, I Volić, Cosimplicial models for spaces of links, J. Homotopy Relat. Struct. 9 (2014) 419-454 MR3258688

[10] D P Sinha, Manifold-theoretic compactifications of configuration spaces, Selecta Math. 10 (2004) 391-428 MR2099074

[11] DP Sinha, Operads and knot spaces, J. Amer. Math. Soc. 19 (2006) 461-486 MR2188133

[12] P A Songhafouo Tsopméné, Formality of Sinha's cosimplicial model for long knots spaces and the Gerstenhaber algebra structure of homology, Algebr. Geom. Topol. 13 (2013) 2193-2205 MR3073913

[13] V Tourtchine, $\mathbf{T}$ Willwacher, Relative (non-)formality of the little cubes operads and the algebraic Schoenflies theorem, preprint (2014) arXiv:1409.0163v1

[14] V Turchin, Hodge-type decomposition in the homology of long knots, J. Topol. 3 (2010) 487-534 MR2684511

[15] V Turchin, Context-free manifold calculus and the Fulton-MacPherson operad, Algebr. Geom. Topol. 13 (2013) 1243-1271 MR3071127

[16] M Weiss, Embeddings from the point of view of immersion theory, I, Geom. Topol. 3 (1999) 67-101 MR1694812

[17] M S Weiss, Homology of spaces of smooth embeddings, Q. J. Math. 55 (2004) 499-504 MR2104688

Institut de Recherche en Mathématique et Physique, Université catholique de Louvain Chemin du Cyclotron 2, B-1348 Louvain-la-Neuve, Belgium

arnaud.songhafouo@uclouvain.be

Received: 15 September 2014 Revised: 1 July 2015 